\title{
ОЦЕНКА ФУНКЦИОНАЛЬНОГО СОСТОЯНИЯ ЩИТОВИДНОЙ ЖЕЛЕЗЫ У БЕРЕМЕННЫХ ЖЕНЩИН В РЕГИОНЕ ЛЕГКОГО ЙОДНОГО ДЕФИЦИТА
}

\author{
Макарова О.Б., Суплотова Л.А.
}

ФГБОУ ВО Тюменский ГМУ Минздрава России

Актуальность заболеваний щитовидной железы во время беременности обусловлена высокой распространенностью патологии и влиянием тиреоидного статуса матери на течение беременности, ее исходы, а также на развитие плода и ребенка. Тюменская область является территорией с легким йодным дефицитом, что также вносит свой вклад в распространенность тиреоидной патологии в регионе.

ЦЕЛЬ: оценить функциональное состояние щитовидной железы у беременных женщин в 1 триместре в регионе легкого йодного дефицита (на примере г.Тюмени).

МАТЕРИАЛЫ И МЕТОДЫ: сплошным методом проведено обследование всех беременных женщин, вставших на учет в женскую консультацию №2 г. Тюмени с июля по октябрь 2019 г. Обследование проводилось после подписания информированного согласия на участие в исследовании, и включало в себя осмотр эндокринолога, проведение ультразвукового исследования щитовидной железы, определение показателей тиреотропного гормона (ТТГ), свободной фракции тироксина (свТ4), антител к тиреопероксидазе (АТ- ТПО). Забор крови выполнялся в 1 триместре, вместе со всеми обследованиями, рекомендованными для постановки на учет в женскую консультацию. Статистическую обработку полученных данных проводили с использованием программных средств пакета Statistica (версия 10).

РЕЗУЛЬТАТЫ И ИХ ОБСУЖДЕНИЕ: всего было обследовано 264 женщины в 1 триместре. Средний возраст беременных составил $29 \pm 5,2$ года (от 18 до 43 лет). Средний срок беременности при обследовании - 9,9 недель. В России в настоящее время нет разработанных референсных значений для разных регионов, поэтому используются нормативы, рекомендованные Европейской тиреоидологической ассоциацией, согласно которым, фиксированным референсным интервалом ТТГ для 1 триместра беременности принят диапазон 0,1 - 2,5 мME/л. У 207 беременных (78,4\%) - показатели ТТГ находились в пределах референсных значений. ТТГ выше 2,5 MME/л определен у 57 женщин (21,5\%), из которых только 16 беременных (6\%) имели уровень выше 4,0 мME/л, и у 1 женщины выявлен манифестный гипотиреоз (0,37\%) с уровнем ТТГ выше 10 мМЕ/л и низким свТ4. Таким образом, большинство женщин с ТТГ выше 2,5 мМЕ/л имели уровни в диапазоне от 2,5 - до 4,0 мMЕ/л (41 - 15,5\%), что сопоставимо с результатами других исследований (П.В. Попова, 2018 г). Субклинический тиреотоксикоз был диагностирован только у 3 беременных, что составило 1,13\%. Повышение титров АТ-ТПО зафиксировано у 28 человек (10,6\%), из них уровень ТТГ выше 2,5 мME/л определен у 21,4\%. Также при анализе группы беременных с эутиреозом - повышение титров АТ-ТПО определено у $10 \%$.

Таким образом, в исследовании показана необходимость включения обследования функционального состояния щитовидной железы всем беременным женщинам, а также необходимость разработки триместр специфических референсных интервалов с учетом проживания в регионе с легким йодным дефицитом. 